\title{
Epigenomics and transcriptomics analyses of multiple system atrophy brain tissue supports a role for inflammatory processes in disease pathogenesis
}

Conceição Bettencourt ${ }^{1,2^{*+}} \mathbb{D}$, Ignazio S. Piras ${ }^{3+}$, Sandrine C. Foti ${ }^{1,4}$, Joshua Talboom ${ }^{3}$, Yasuo Miki, ${ }^{1,5}$ Tammaryn Lashley ${ }^{1,4}$, Robert Balazs ${ }^{1,4}$, Emmanuelle Viré ${ }^{6}$, Thomas T. Warner ${ }^{1,2,7}$, Matt J. Huentelman ${ }^{3+}$ and Janice L. Holton ${ }^{1,2 \dagger}$

Keywords: Methylomics, DNA methylation, EWAS, Transcriptomics, Gene expression, RNAseq, MSA,

Neurodegeneration, Inflammation

To the Editors,

Multiple system atrophy (MSA) is a fatal neurodegenerative disease and its aetiology remains elusive. The pathological hallmark of MSA is the presence of glial cytoplasmic inclusions (GCIs) containing fibrillar $\alpha$ synuclein in oligodendrocytes $[3,6]$, but the regional vulnerability of the brain to these GCIs remains poorly understood. We read with interest the paper by Rydbirk and colleagues recently published in Acta Neuropathologica Communications [5]. They investigated DNA methylation $(5 \mathrm{mC})$ and hydroxymethylation $(5 \mathrm{hmC})$ changes in prefrontal cortex samples from MSA patients. We reported previously [1] total DNA methylation $(5 \mathrm{mC}+5 \mathrm{hmC})$ changes in MSA, and this work by Rydbirk et al. [5] further supports a contribution of epigenetic factors, namely DNA methylation, to MSA brain pathophysiology.

* Correspondence: c.bettencourt@ucl.ac.uk

${ }^{\dagger}$ Matt J. Huentelman and Janice L. Holton joint senior authors

${ }^{1}$ Queen Square Brain Bank for Neurological Disorders, UCL Queen Square Institute of Neurology, London, UK

${ }^{2}$ Department of Clinical and Movement Neurosciences, UCL Queen Square Institute of Neurology, Queen Square Brain Bank for Neurological Disorders, 1 Wakefield street, London WC1N 1PJ, UK

Full list of author information is available at the end of the article
Given the differentially methylated CpGs (e.g. in AREL1 and KTN1), regions (DMRs) and blocks reported for the $5 \mathrm{mC}$ fraction by Rydbirk and colleagues [5], we performed additional loci-specific analysis of our MSA DNA methylation data [1]. We used data from our discovery cohort [1], which was composed of neuropathologically confirmed MSA mixed cases and controls, and investigated multiple brain regions characterized by different degrees of GCI burden in MSA, including the cerebellum, and the frontal and occipital lobes. In the frontal lobe, no changes were detected in AREL1 nor in the reported intergenic CpGs (Supplementary Table S1.1). Although with small effects (absolute delta betas $<5 \%$ ), two CpGs in KTN1 were nominally significant (cg14002714 and cg21059882; $p<0.05$ ). Regarding the block covering PHF3, two CpGs were nominally significant (cg16049132 and cg10435600; $p<0.05$ ). Additionally, $52 \mathrm{CpGs}$ in the DMR genes were nominally significant in our data, 14 of which (in 5 genes: FUT4, BCAR1, CTSZ, ZIC4 and FERMT3) demonstrated absolute delta betas of $\geq 5 \%$. With the exception of cg18023065 in FUT4, none of these changes passed multiple testing correction $\left(p<9.07 \times 10^{-5} \quad[0.05 / 551\right.$ CpGs]). Some of those CpGs and additional CpGs were also nominally significant in the other brain regions 
analysed (Supplementary Table S1.1). Interestingly, the DMR in the FUT4/PIWIL4 promoter (chromosome 11: 94278407-94,279,068), replicates a DMR we found in the frontal lobe and cross-region analyses of our previous study (Supplementary Tables S3.1 and S3.3 from [1]).

The study by Rydbirk et al. [5] and ours [1] have markedly different designs: a) Rydbirk et al. [5] included white and grey matter from the frontal lobe, whilst we carefully dissected white matter to enrich for oligodendrocytes and analysed different brain regions; and b) they investigated the contributions of $5 \mathrm{mC}$ and $5 \mathrm{hmC}$ separately. We are aware that in our data alterations in the $5 \mathrm{mC}$ and $5 \mathrm{hmC}$ proportions can counteract each other and mask the detection of significant changes in total methylation. As an example, the AREL1 shift from $5 \mathrm{mC}$ to $5 \mathrm{hmC}$ reported by Rydbirk et al. [5] is masked in our total DNA methylation data, highlighting an advantage of analysing $5 \mathrm{mC}$ and $5 \mathrm{hmC}$ separately. In addition, distinct cell type compositions in the brain tissue samples may contribute to discordant findings. According to RNAseq data from major brain cell types (data from Zhang et al. [7], Supplementary Fig. 1), our results support methylation changes in genes that are highly expressed in oligodendrocytes, including KTN1 and $P H F 3$, or more highly expressed in microglia/macrophages, including FUT4, CTSZ, and FERMT3 (Supplementary Fig. 1). Conversely, genes more highly expressed in neurons and/or with low expression in oligodendrocytes, such as AREL1, were less susceptible to DNA methylation changes in our dataset.

Findings from Rydbirk et al. [5] also report increased AREL1 and MHC class I HLA gene expression in MSA brains. We therefore investigated in our MSA cerebellar white matter RNAseq data [4] gene expression changes in all of the genes reported by Rydbirk et al. [5]. Our study includes two independent cohorts of 66 MSA and 66 healthy controls and laser captured oligodendrocytes [4]. Although we did not find differential expression for AREL1 or PHF3, we found a nominally significant $(p<$ $0.05)$ downregulation of KTN1 $(\log 2 \mathrm{FC}=-0.465)$, and upregulation of CTSZ $(\log 2 \mathrm{FC}=0.817), N C S 1 \quad(\log 2$ $\mathrm{FC}=0.813)$ and $Z I C 4(\log 2 \mathrm{FC}=1.520)$ in some groups of our cohort 1 (Supplementary Table S2). The upregulation of ZIC4 was replicated in cohort 2 of our study $(\log 2 \mathrm{FC}=1.551)$ and remained significant when accounting for multiple testing adjustments in the combined analysis of both cohorts 1 and $2(\log 2 \mathrm{FC}=1.536$; adjusted- $p=0.022$ ). In our RNAseq data, MHC class I HLA genes have shown inconsistent results across cohorts/groups, with only $H L A-A$ showing nominally significant upregulation in one group of cohort 1 (log2 $\mathrm{FC}=1.156$ in MSA-P; $p=0.002)$ and HLA-F in oligodendrocytes $(\log 2 \mathrm{FC}=1.982 ; p=0.032)$. Some of the MHC class I HLA genes, including $H L A-A$, have also shown nominally significant DNA methylation changes in our data (Supplementary Table S1.2).

Overall, we consider that these recent studies by Bettencourt et al. [1], Piras et al. [4], and Rydbirk et al. [5] are complementary, and bring important insights into the brain pathophysiology of MSA. All show changes in DNA methylation or in gene expression levels of genes that are more highly expressed in microglia/macrophages, therefore supporting previous studies highlighting the involvement of inflammatory processes in MSA (e.g. [2]).

\section{Supplementary information}

Supplementary information accompanies this paper at https://doi.org/10. 1186/s40478-020-00946-1.

Additional file 1: Supplementary Table S1.1. Loci-specific analysis of differentially methylated CpGs, regions and blocks identified by Rydbirk et al. 2020. Supplementary Table S1.2. Loci-specific analysis of differential methylation in MHC class I HLA genes.

Additional file 2: Supplementary Table S2. Loci-specific analysis of cerebellar white matter gene expression for genes reported to be differentially methylated and/or differentially expressed by Rydbirk et al. 2020.

Additional file 3: Supplementary Figure 1. Boxplots of normalized counts in different brain cell types [7] for the most relevant genes detected in $[1,5]$. Raw data were downloaded from Sequencing Reads Archive (\#SRP064454), pseudoalignment was conducted with Kallisto v0.46.1, and counts were normalized with DESeq2 v1.26.0. Data were from hippocampus, temporal lobe and fetal cortex. Tumor samples were excluded from the dataset. FA: fetal astrocytes. A: astrocytes. N: neurons. O: oligodendrocytes. M: microglia. E: endothelial cells.

\section{Abbreviations}

DMRs: Differentially methylated regions; FC: Fold change; GCls: Glial cytoplasmic inclusions; MSA: Multiple system atrophy; MSA-C: Clinical subtype of multiple system atrophy with a predominant cerebellar phenotype; MSA-P: Clinical subtype of multiple system atrophy with a predominant parkinsonian phenotype; RNAseq: Whole-transcriptome data obtained by next-generation sequencing

\section{Acknowledgements}

The authors would like to thank Ms. Gaganjit Kaur Madhan (MSc) for sample processing and the UCL Genomics centre for advice and processing of the EPIC arrays. Geidy Serrano, Lucia Sue, and Thomas Beach for providing MSA case and control tissue, and Christiane Bleul, Isabelle Schrauwen, Lorida Llaci, Matthew De Both, and Marcus Naymik for aiding in the generation and analysis of the RNA data.

\section{Authors' contributions}

$\mathrm{CB}$ contributed to the design of the work, analysis and interpretation of data, and drafted the work; ISP contributed to the analysis and interpretation of data, and critically revised the work; SCF and JT contributed to the generation of the data. YM revised clinical and pathological data. EV, RB and $\mathrm{TL}$ have contributed for the design of the work, interpretation of the data, and critically revised the work; TTW made financial contributions to enable the work, and critically revised the work; MJH and JLH made substantial contributions to the conception and supervision of the work, and critically revised the work. All authors have approved the submitted version.

\section{Funding}

Queen Square Brain Bank for Neurological Disorders receives support from the Reta Lila Weston Institute of Neurological Studies and the Medical Research Council (MRC). CB is supported by the Multiple System Atrophy Trust, the British Neuropathological Society, and an Alzheimer's Research UK 
Research Fellowship. TL is supported by an Alzheimer's Research UK Senior Fellowship. TTW is supported by the Reta Lila Weston Trust and the MRC (N013255/1). JLH is supported by the Multiple System Atrophy Trust; the Multiple System Atrophy Coalition; Fund Sophia, managed by the King Baudouin Foundation and Karin \& Sten Mortstedt CBD Solutions. This research was supported by the National Institute for Health Research University College London Hospitals Biomedical Research Centre. The authors also acknowledge funding support from the NIH National Institute of Neurological Disorders and Stroke (R21-NS093222 to MJH), Tissue from The Brain and Body Donation Program at the Banner Sun Health Research Institute is supported by the National Institute of Neurological Disorders and Stroke (U24 NS072026 National Brain and Tissue Resource for Parkinsons Disease and Related Disorders), the National Institute on Aging (P30 AG19610 Arizona Alzheimers Disease Core Center), the Arizona Department of Health Services (contract 211002, Arizona Alzheimers Research Center), and the Arizona Biomedical Research Commission (contracts 4001, 0011, 05901 and 1001 to the Arizona Parkinson's Disease Consortium).

\section{Availability of data and materials}

Data generated during this study are included in this published article and its supplementary information files. The datasets used for the analyses during the current study available from the corresponding author on reasonable request.

\section{Ethics approval and consent to participate}

All brain tissue from brains donated to the Queen Square Brain Bank (QSBB) archives (UK), including the tissue used for the DNA methylation study, is stored under a licence from the Human Tissue authority (No. 12198). The QSBB brain donation programme and protocols have received ethical approval for donation and research by the NRES Committee London Central [18/LO/0721]. For the RNAseq study, conducted in the USA, all human samples were derived from autopsy tissue and were classified as non-human subject research material according to local regulations.

\section{Consent for publication}

Not applicable.

\section{Competing interests}

The authors declare that they have no conflict of interest.

\section{Author details}

'Queen Square Brain Bank for Neurological Disorders, UCL Queen Square Institute of Neurology, London, UK. ${ }^{2}$ Department of Clinical and Movement Neurosciences, UCL Queen Square Institute of Neurology, Queen Square Brain Bank for Neurological Disorders, 1 Wakefield street, London WC1N 1PJ, UK. ${ }^{3}$ Neurogenomics Division, Translational Genomics Research Institute, Phoenix, AZ, USA. ${ }^{4}$ Department of Neurodegenerative Disease, UCL Queen Square Institute of Neurology, London, UK. ${ }^{5}$ Department of Neuropathology, Institute of Brain Science, Hirosaki University Graduate School of Medicine, Hirosaki, Japan. ${ }^{6}$ Institute of Prion Diseases, MRC Prion Unit at UCL, Courtauld Building, 33 Cleveland Street, London, UK. ${ }^{7}$ Reta Lila Weston Institute of Neurological Studies, UCL Queen Square Institute of Neurology, London, UK.

Received: 6 April 2020 Accepted: 7 May 2020

Published online: 14 May 2020

\section{References}

1. Bettencourt C, Foti SC, Miki Y, Botia J, Chatterjee A, Warner TT, Revesz T, Lashley T, Balazs R, Vire E et al (2020) White matter DNA methylation profiling reveals deregulation of HIP1, LMAN2, MOBP, and other loci in multiple system atrophy. Acta Neuropathol 139:135-156. https://doi.org/10. 1007/s00401-019-02074-0

2. Kiely AP, Murray CE, Foti SC, Benson BC, Courtney R, Strand C, Lashley T, Holton $J$ (2018) Immunohistochemical and molecular investigations show alteration in the inflammatory profile of multiple system atrophy brain. J Neuropathol Exp Neurol 77:598-607. https://doi.org/10.1093/jnen/nly035

3. Papp MI, Kahn JE, Lantos PL (1989) Glial cytoplasmic inclusions in the CNS of patients with multiple system atrophy (striatonigral degeneration, olivopontocerebellar atrophy and shy-Drager syndrome). J Neurol Sci 94:79-100
4. Piras IS, Bleul C, Schrauwen I, Talboom J, Llaci L, De Both MD, Naymik MA, Halliday G, Bettencourt C, Holton JL et al (2020) Transcriptional profiling of multiple system atrophy cerebellar tissue highlights differences between the parkinsonian and cerebellar sub-types of the disease. bioRxiv https:// www.biorxiv.org/content/10.1101/2020.02.11.944306v1. Accessed 12 Feb 2020.

5. Rydbirk R, Folke J, Busato F, Roche E, Chauhan AS, Lokkegaard A, Hejl AM, Bode M, Blaabjerg M, Moller M et al (2020) Epigenetic modulation of AREL1 and increased HLA expression in brains of multiple system atrophy patients. Acta Neuropathol Commun 8:29. https://doi.org/10. 1186/s40478-020-00908-7

6. Spillantini MG, Crowther RA, Jakes R, Cairns NJ, Lantos PL, Goedert M (1998) Filamentous alpha-synuclein inclusions link multiple system atrophy with Parkinson's disease and dementia with Lewy bodies. Neurosci Lett 251:205-208

7. Zhang Y, Sloan SA, Clarke LE, Caneda C, Plaza CA, Blumenthal PD, Vogel H, Steinberg GK, Edwards MS, Li G et al (2016) Purification and characterization of progenitor and mature human astrocytes reveals transcriptional and functional differences with mouse. Neuron 89:37-53. https://doi.org/10. 1016/j.neuron.2015.11.013

\section{Publisher's Note}

Springer Nature remains neutral with regard to jurisdictional claims in published maps and institutional affiliations.

\section{Ready to submit your research? Choose BMC and benefit from:}

- fast, convenient online submission

- thorough peer review by experienced researchers in your field

- rapid publication on acceptance

- support for research data, including large and complex data types

- gold Open Access which fosters wider collaboration and increased citations

- maximum visibility for your research: over $100 \mathrm{M}$ website views per year

At BMC, research is always in progress.

Learn more biomedcentral.com/submissions 